not examination of the one alternate therapy most likely to explain discontinuation rates of ACE inhibitors, the most logical therapeutic alternative to ACE inhibitors, the angiotensin II receptor blockers (ARBs).

For the other 2 drug classes studied, proton-pump inhibitors (PPIs) and statins, the results of implementation of 3-tier copay designs were inconsistent among the 2 employer groups and their matched comparisons. There was a higher rate of discontinuation of statins for employer 1 versus its comparison $(P=0.04)$ but not for employer 2 and its comparison $(P=0.45)$. There was a higher rate of discontinuation of PPIs for employer 1 versus its comparison $(P<0.001)$ but not for employer 2 versus its comparison $(P=0.79)$. Part of the reason for these apparently disparate findings may lie with the magnitude of the copays and the type of change. Beneficiaries of the employer 1 drug plan were converted from a single-tier copay ( $\$ 7$ for generic or brand-name drug for community pharmacy) to a 3-tier copay design (community pharmacy copayments of $\$ 8$ for generic, $\$ 15$ for preferred brand drugs and $\$ 30$ for nonpreferred brand drugs) compared with a comparison group with an unchanged 2-tier plan of $\$ 8$ copay for generic drugs and $\$ 15$ copay for brand-name drugs. Beneficiaries in employer 2 were confronted with a smaller magnitude and extent of change, transitioned from a 2-tier plan of $\$ 6$ copay for a generic drug and $\$ 12$ for a brand-name drug to a 3-tier plan design with the same copays for tier- 1 and tier- 2 drugs and simply the addition of a third-tier copay of $\$ 24$ for nonpreferred drugs. The comparison group for employer 2 had an unchanged 2-tier plan design of $\$ 6$ and $\$ 12$. Some consumer research suggests that copay differences of as much as $\$ 35$ per prescription are necessary to influence consumer behavior in use of PPIs, ${ }^{16}$ a dollar amount more than twice the tier-3 copayment "penalty" in the 3-tier drug benefit designs in this study of PPIs, ACE inhibitors, and statin drugs.

So, there is much that remains to be answered about the effects of 3-tier drug benefit copay designs on clinical, service-humanistic, and cost outcomes. The important and useful answers will come from carefully designed studies, but even these results will require interpretation since drug formularies upon which 3-tier copay plans are based differ markedly in content from one managed care organization to another. For example, the inconsistent and even contradictory results in the recent study might be explained, in part, by characteristics unique to the study population. The formulary of the pharmacy benefit manager (PBM) contained only 2 statins in tier 3 throughout the study, fluvastatin and lovastatin, and the latter became available by generic name in the middle of the study period. Readers might be better able to judge the importance of this variable if the authors had disclosed the actual patient counts for each of the 3 statin drugs in tier-3, one of which (Mevacor) became available by generic name in December 2000, the midpoint of the study period, and cerivastatin (Baycol), which changed tier-copay status from nonformulary (tier-3 copay) to formulary status (tier-2 copay) in October 2000, also in the midpoint of the study period. Baycol was withdrawn from the market in August 2001, about 5 months prior to the completion of the postintervention study period and 10 months after changing from nonformulary (tier-3 copay) to formulary (tier-2 copay) status for this PBM.

Due, in part, to the content of the tier-3 (nonformulary) statin drugs for this PBM, the utilization of tier-3 statin drugs in this study population was exceptionally low and not generalizable to other patient populations in drug benefit plans. The highest utilization of tier-3 statin drugs was found not in the comparison groups but, ironically, in the intervention (3-tier copay) groups, 3.5\% of 933 patients for employer 2 and $3.4 \%$ of 2,608 statin patients for employer 1 versus $3.1 \%$ of 3,391 statin patients for the comparison group to employer 1 and $1.7 \%$ of the comparison group to employer 2. Therefore, measurement of the effect of the tier-3 plan design change on discontinuation of statin drugs was undertaken on a very small number of patients: 3 (9.1\%) discontinued statin therapy versus 1 patient (4.0\%) for the comparison group $(P=0.45)$ and 19 patients (21.3\%) for employer 1 versus 11 patients (10.6\%) for the comparison group $(P=0.04)$.

Other nuances further complicated the comparisons for the 3 drug classes in this study. In addition to the dual-tier copay status for lovastatin (tier-3 copay [Mevacor] and tier- 1 copay effective December 2000) and the switch in copay for Baycol, followed by its withdrawal from the market, for the PPIs, esomeprazole was a tier-3 PPI before November 2001 but changed to a tier-2 copay in the latter part of the postintervention period, on November 1 , 2001. For the ACE inhibitors, captopril was both a tier- 1 copay drug and a tier-2 (Capoten) copay drug (and most MCOs would place the multiple-source brand drug Capoten in tier-3).

Nevertheless, this recent study of the effects of 3-tier drug copay designs for 2 employers provides a valuable reminder that the answers to the important questions regarding tier-copay drug benefit designs will depend in significant part upon how we ask the questions. It should come as no surprise that neither the answers nor the questions are simple. Behavioral change, including discontinuation rates, will likely depend upon the magnitude of the financial incentive(s) as well as the degree of change from the previous design to the new drug benefit design. When interpreting the results of research conducted on the effects of multi-tier drug benefit plan designs, readers, at a minimum, should assess 2 key variables: (a) the content of the drug formulary tiers and (b) the dollar magnitude of the financial incentives/penalties and the degree of change that are imposed by the new benefit design.

\section{Managed Care Pharmacy- Weighing Clinical, Service, and Cost Outcomes}

The creation of drug benefit plan designs that result in improvement in clinical, service-humanistic, and cost outcomes requires competent and experienced design architects. Managed care pharmacists possess unique knowledge for this task-the combination of information gleaned from clinical trials, experience with assessment of service outcomes, and the ability to identify precisely the price per day of drug therapy. Admittedly, better knowledge of total cost (e.g., direct drug and 
medical costs and indirect costs) is needed, but managed care pharmacists can identify precisely the actual net cost per day of drug therapy among alternate products used for the same indication. ${ }^{17}$ A recent survey of 100 pharmacy and medical directors in health plans found that $78 \%$ of the plans that preferred pantoprazole did so because it was the low-price leader among the protonpump inhibitors (PPIs). ${ }^{18}$ This fact reflects an opinion among pharmacy and medical directors that the 5 PPIs are relatively equivalent in safety and efficacy, and the preference in formulary position defers to price, for which pantoprazole at the time of the survey had a clear and distinct advantage over the 4 competing agents, including generic omeprazole.

\section{Drug Formulary Decisions-Evaluating the Risks and Benefits of Hormone Replacement Therapy}

A randomized controlled trial (RCT) found that $0.625 \mathrm{mg}$ of estrogen plus $5 \mathrm{mg}$ medroxyprogesterone hormone replacement therapy (HRT), was approximately 2 times as effective as $5 \mathrm{mg}$ of alendronate in preserving or building bone mineral density in postmenopausal women. ${ }^{19}$ The favorable effect of HRT on mineral density is manifest in dental bone as well as skeletal bone. A review of the medical literature found 1,518 articles on estrogen and osteoporosis, of which 20 were deemed relevant to the subject of HRT on mandibular bone or other dental bone effects of HRT and clinical or cost outcomes. The 20 studies involved data collection for 13,735 women for whom dental, HRT, or osteoporosis outcomes were collected, and the conclusion was that HRT has favorable effects on dental outcomes and dental costs. ${ }^{20}$

The favorable effects of HRT in osteoporosis appear to be offset by adverse effects in breast cancer. The results from the HRT portion of the Women's Health Initiative (WHI) RCT was announced on July 9, 2002. This study enrolled 16,608 postmenopausal women without coronary heart disease (CHD) and randomized them to combination HRT or placebo. The study was designed to examine the effect of combination HRT on the prevention of heart disease and hip fractures and to identify any associated risk for breast and colon cancer. Originally, the study was to continue until 2005, but the data safety monitoring board stopped the trial after finding that the risk of therapy began to outweigh the benefit when they analyzed accumulated data at the end of May 2002. With an average of 5.2 years of follow-up (a range of 3.5 years to 8.5 years), researchers found a higher rate of invasive breast cancer in the treatment arm: 38 versus 30 cases of invasive breast cancer per 10,000 person-years. Any prior use of HRT was associated with 114 cases of breast cancer over the average 5.2 years of follow-up, (a rate of 1.34\%), compared with 102 cases (a rate of $1.26 \%$ ) for women who had never taken HRT. In other words, WHI found that HRT was associated with 8 additional cases of breast cancer per 10,000 women treated per year.

The results from the Heart and Estrogen/progestin Replacement Study (HERS), HERSII, ${ }^{21} \mathrm{WHI},{ }^{22}$ and Womens' Estrogen LipidLowering Hormone Atherosclerosis Regression Trial (WELLHART ${ }^{23}$ suggest that combination estrogen and progestin is not associated with favorable effects on the progression of atherosclerosis or the prevention of CHD events. In WHI, HRT was associated with increases in CHD events (29\%; 37 versus 30 per 10,000 person-years; most of the excess was in nonfatal myocardial infarction [MI] with no significant differences in CHD deaths or revascularization procedures [coronary artery bypass graft and percutaneous transluminal coronary angioplasty], stroke [41\%; 29 versus 21 events per 10,000 person-years; most of the difference in nonfatal stroke], and pulmonary embolism [a doubling of the rate in the placebo group]). On the positive side, combination HRT was shown to result in 37\% fewer cases of colon cancer (10 versus 16 per 10,000 person-years), 33\% fewer hip fractures (10 versus 15 per 10,000 person-years), 24\% fewer fractures overall, and 23\% fewer osteoporotic fractures. There was no difference in mortality between the 2 groups. It should be noted that the overall risk of the negative events was quite low (i.e., an individual's risk of any of the events was less than $0.1 \%$ ); however, from a population basis, there is significant risk relative to minor benefits. For a small subset of women with CHD (who would have been eligible for HERS; i.e., prior MI or revascularization procedure; $\mathrm{N}=400$ ), there were 19 CHD events versus 16 events (hazard ratio 1.28), and, for the remaining women, the identical hazard ratio of $1.28,145 \mathrm{CHD}$ events for HRT versus 106 CHD events for placebo. Researchers concluded that the use of combination HRT should not be initiated or continued for the primary prevention of $\mathrm{CHD}$. Also noteworthy, $42 \%$ of HRT users and 38\% of placebo users stopped taking study drugs at some time. ${ }^{24}$

Others have helped explain the sometimes divergent data on postpmenopausal HRT from the more than 50 observational studies and RCTs, ${ }^{25}$ one with a table that compares the findings from WHI versus HERS versus observational studies of estrogen with progestin in 6 key outcomes (breast cancer, colorectal cancer, hip fracture, stroke, pulmonary embolism and CHD) ${ }^{26} \mathrm{HRT}$, while relatively inexpensive, may not be the best choice for osteoporosis. Estrogen alone may be effective in primary prevention of cardiovascular disease (CVD) but may not be effective in secondary prevention (in women with established CHD). Estrogen or HRT may help retard memory loss in women and might prevent Alzheimer's disease. ${ }^{27}$ HRT might increase the risk of breast cancer, but the cancers associated with HRT may be more benign tumors, possibly explaining the observation of lower breast cancer mortality rates among HRT users compared with nonusers. Estrogen unopposed by progestin has a more favorable effect on high-density lipoprotein (HDL), ${ }^{28}$ but micronized progesterone in combination with estrogen maintains the benefits of estrogen on HDL, ${ }^{29}$ and unopposed estrogen has been associated with favorable cardiovascular effects. ${ }^{30,31}$ Estrogen alone may not be associated with an increase in breast cancer risk. ${ }^{32}$ Research on clinical outcomes of estrogen alone continues in WHI and elsewhere, and, after nearly 20 years of research on estrogen and HRT, definitive answers remain elusive, in part due to the high ratio of subjects lost to follow-up in RCTs such as WHI. ${ }^{33}$ However, the available information at this time suggests that there may be significant differences in outcomes 